\title{
Analytical validation and chromosomal distribution of regions of homozygosity by oligonucleotide array comparative genomic hybridization from normal prenatal and postnatal case series
}

\author{
Jiadi Wen ${ }^{1 *}$ (D) Kathleen Comerford ${ }^{2}$, Zhiyong $\mathrm{Xu}^{3}$, Weiqing $\mathrm{Wu}^{3}$, Katherine Amato ${ }^{1}$, Brittany Grommisch',
} Autumn DiAdamo ${ }^{1}$, Fang $\mathrm{Xu}^{4}$, Hongyan Chai ${ }^{1}$ and Peining $\mathrm{Li}^{1^{*}}$

\begin{abstract}
Background: Regions of homozygosity $(\mathrm{ROH})$ are continuous homozygous segments commonly seen in the human genome. The integration of single nucleotide polymorphism (SNP) probes into current array comparative genomic hybridization $(\mathrm{aCGH})$ analysis has enabled the detection of the $\mathrm{ROH}$. However, for detecting and reporting biologically relevant $\mathrm{ROH}$ in a clinical setting, it is necessary to assess the analytical validity of SNP calling and the chromosomal distribution of $\mathrm{ROH}$ in normal populations.

Methods: The analytical validity was evaluated by correlating the consistency of SNP calling with the quality parameters of aCGH and by accessing the accuracy of SNP calling using PCR based restriction enzyme digestion and Sanger sequencing. The distribution of $\mathrm{ROH}$ was evaluated by the numbers, sizes, locations, and frequencies of $\mathrm{ROH}$ from the collection of data from parental, postnatal, and prenatal case series that had normal aCGH and chromosome results.

Results: The SNP calling failure rate was 20-30\% with a derivative Log2 ratio (DLR) below 0.2 and increased significantly to $30-40 \%$ with DLR of $0.2-0.4$. The accuracy of SNP calling is $93 \%$. Of the 958 cases tested, $34 \%$ had no $\mathrm{ROH}, 64 \%$ had one to four $\mathrm{ROH}$, and less than $1 \%$ had more than five $\mathrm{ROH}$. Of the $1196 \mathrm{ROH}$ detected, $95 \%$ were less than $10 \mathrm{Mb}$. The distribution of numbers and sizes of ROH showed no differences among the parental, pediatric and prenatal case series and test tissues. The chromosomal distribution of $\mathrm{ROH}$ was non-random with $\mathrm{ROH}$ seen most frequently in chromosome 8, less frequently in chromosomes 2, 6, 10,12, 11 and 18, and most rarely seen on chromosomes 15, 19, 21 and 22. Recurrent $\mathrm{ROH}$ occurring with a frequency greater than 1\% were detected in 17 chromosomal loci which locates either in the pericentric or interstitial regions.

Conclusion: With a quality control parameter of DLR set at below 0.2 , the consistency of SNP calling would be $75 \%$, the accuracy of SNP call could be $93 \%$, and the observed chromosomal distribution of ROH could be used as a reference. This aCGH analysis could be a reliable screening tool to document biologically relevant $\mathrm{ROH}$ and recommend further molecular analysis.
\end{abstract}

Keywords: Regions of homozygosity (ROH), Single nucleotide polymorphism (SNP) calling, Array comparative genomic hybridization $(\mathrm{aCGH})$

\footnotetext{
*Correspondence: jiadi.wen@yale.edu; peining.li@yale.edu

'Department of Genetics, Yale University School of Medicine, New Haven, CT 06520, USA

Full list of author information is available at the end of the article
}

(c) The Author(s). 2019 Open Access This article is distributed under the terms of the Creative Commons Attribution 4.0 International License (http://creativecommons.org/licenses/by/4.0/), which permits unrestricted use, distribution, and reproduction in any medium, provided you give appropriate credit to the original author(s) and the source, provide a link to the Creative Commons license, and indicate if changes were made. The Creative Commons Public Domain Dedication waiver (http://creativecommons.org/publicdomain/zero/1.0/) applies to the data made available in this article, unless otherwise stated. 


\section{Background}

Copy number neutral segments showing continuous homozygosity with no intervening heterozygosity, termed 'regions of homozygosity' (ROH) or sometimes 'runs of homozygosity', were first identified using the denser genome-wide microsatellite scans by Broman in 1999 [1]. Other terms used in the literature included 'long contiguous stretches of homozygosity' which is interchangeable to $\mathrm{ROH}$ and 'loss of heterozygosity' or 'absence of heterozygosity' which could be copy number neutral or a deletion occurring as a somatic event in cancer [2]. $\mathrm{ROH}$ arise when identical haplotypes are inherited from each parent and thus a long tract of genotypes is homozygous. These autozygous segments may represent uniparental disomy (UPD), ancestral homozygosity due to linkage disequilibrium, or regions inherited from a more recent common ancestor that are identical by descent (IBD). Cousin marriage or inbreeding gives rise to such autozygosity. The detection of large ROH, UPD, and consanguinity by the presence of multiple $\mathrm{ROH}$ could have diagnostic implications in clinical laboratories performing single nucleotide polymorphism (SNP) microarray analysis $[3,4]$. Genome-wide data reveal that $\mathrm{ROH}$ are universally common in human genomes even among outbred individuals $[1,5]$. The numbers and sizes of $\mathrm{ROH}$ are believed to be a reflection of recombination rates and individual demographic history, while the homozygosity burden can be used to investigate the genetic architecture of complex diseases $[6,7]$.

Over the past decade, the validation and utilization of genomic microarray platforms in clinical cytogenetics has significantly increased the analytical resolution and diagnostic yield in prenatal and postnatal genetic evaluations $[8,9]$. Subsequently, microarray analysis has been recommended as the first-tier genetic test for patients with developmental disabilities or congenital anomalies [10]. Today, microarray analyses using either array comparative genomic hybridization $(\mathrm{aCGH})$ with copy number and SNP oligonucleotide probes or SNP genotyping microarray provide a genome-wide analysis at a kilo-base level resolution for the detection of copy number variants $(\mathrm{CNV})$ and $\mathrm{ROH}[4,10-15]$. To detect and report biologically relevant $\mathrm{ROH}$ in a clinical setting, it is necessary to validate the technical procedures and understand the distribution of $\mathrm{ROH}$ in the outbred population. Before the publication of the ACMG guideline [16], the reporting practices among laboratories were highly variable due to the differences among testing platforms, threshold parameters, and laboratory policies. Factors such as the probe density, the probe distribution across the genome, and the quality of the SNP calling (including error rates) influenced the quality of $\mathrm{ROH}$ detection. Each laboratory interpreted results of homozygosity with their own discretion per the conflicting data in the literature regarding what cutoff level should be used $[6,17]$. In this study, we assessed the analytical validity of Agilent's oligonucleotide aCGH on detecting $\mathrm{ROH}$ by evaluating the consistency and accuracy of SNP calling. We also analyzed the numbers, sizes, locations, and frequencies of $\mathrm{ROH}$ from prenatal and postnatal normal case series. These results provided an important reference in detecting and reporting $\mathrm{ROH}$ findings for a better diagnostic practice to the patients.

\section{Material and methods}

\section{Patient population}

The $\mathrm{ROH}$ data were collected from aCGH studies performed on prenatal and postnatal case series at the Yale Clinical Cytogenetics Laboratory from January 2014 to December 2017 [18]. All cases showed normal aCGH and chromosome results and were considered to be 'cytogenomically normal' individuals. Four case series were organized based on prenatal or postnatal clinical indications and tested sample types. The normal parental case series included 142 healthy adults who were tested in a follow up parental study to rule out carriers of a balanced chromosomal rearrangement and to verify familial origin of variants of uncertain significance detected in their children. The pediatric case series included 500 cases with clinical indications of developmental/intellectual delay, autism spectrum disorders, intrauterine growth retardation (IUGR), multiple congenital anomalies, hearing loss, seizures, and other constitutional conditions. The aCGH and chromosome analysis were performed on peripheral blood specimens for parental and pediatric case series. The prenatal case series included 195 cases performed on chorionic villi samples (CVS) and 121 cases on amniotic fluid (AF) samples. Clinical indications for prenatal studies included advanced maternal age ( $>35$ years), suspected fetal anomaly, previous abnormal pregnancies and a family history of a hereditary disease. The parental, pediatric, prenatal CVS, and prenatal AF case series represented a full spectrum of constitutional cytogenomic analysis in the current clinical settings.

Additional criteria for data selection included the Derivative Log2 Ratio (DLR) spread and $\mathrm{ROH}$ size limitations. $\mathrm{ROH}$ data was collected only from cases that had a DLR equal to or less than 0.2, except for cases used for the analysis of SNP calling failure rate, in which the cases with the different range of DLR $(\leq 0.2,0.2-0.3, \geq$ $0.3)$ were included. The average SNP probes per megabase $(\mathrm{Mb})$ genome is 19 probes, the arbitrary size cutoff of $\mathrm{ROH}$ is $1 \mathrm{Mb}$. Therefore, any $\mathrm{ROH}$ less than $1 \mathrm{Mb}$ was excluded. This study also excluded cases with multiple $\mathrm{ROH}$ with a sum size greater than $3 \%$ of the genome size that could indicate a family relationship.

\section{DNA extraction and aCGH analysis}

DNA was extracted from peripheral blood specimens, CVS and cultured amniocytes using the Gentra Puregene 
Kit (Qiagen, Valencia, CA) following standardized procedures adopted from the manufacturer's instructions. The oligonucleotide aCGH analyses using Agilent's SurePrint G3 Human Genome CGH + SNP microarray $180 \mathrm{~K}$ kit or the $400 \mathrm{~K}$ kit (Agilent Technologies, Inc., Santa Clara, CA) were performed as previously described [8]. The Agilent $180 \mathrm{~K}$ kit contains 110,712 CGH probes and 59,647 SNP probes, and the $400 \mathrm{~K}$ kit contains 292,097 CGH probes and 119,091 SNP probes (2 probes/SNP). The spatial resolution is an average of approximately 19 SNP probes per $\mathrm{Mb}$ of genome. The peripheral blood samples from parental and pediatric cases were analyzed with either the $400 \mathrm{~K}$ or the $180 \mathrm{~K}$ kit, and all prenatal samples (CVS and AF) were analyzed using the $180 \mathrm{~K}$ kit. This aCGH procedure can achieve $99 \%$ sensitivity and $99 \%$ specificity using a sliding window of five to seven contiguous oligonucleotides, indicating an analytical resolution of $100-150 \mathrm{~Kb}$ for the $180 \mathrm{~K}$ kit and $40-50 \mathrm{~Kb}$ for the $400 \mathrm{~K}$ kit [8]. The aCGH data were processed through the Agilent CytoGenomics Software to generate a report of detected $\mathrm{CNV}$ and $\mathrm{ROH}$ with genomic and chromosomal visualization and genomic coordinates following the UCSC genome browser GRCH37/hg19 assembly (http:// genome.ucsc.edu/).

\section{Assessing the consistency and accuracy of SNP calling by aCGH}

The readout of SNP alleles by Agilent Cytogenomics Software includes successful calling of homozygous and heterozygous alleles and failure calling of 'NN'. The SNP calling failure rate was calculated by the number of ' $\mathrm{NN}$ ' alleles divided by the total number of SNPs. It is thought that the SNP calling can be affected by the quality of fluorescent labeling of test and control DNAs and co-hybridization them onto the microarray. DLR from the test and control hybridization signals has been used as an important quality control parameter for aCGH. To assess the consistency of SNP calling, 30 cases each analyzed by $180 \mathrm{~K}$ and $400 \mathrm{~K}$ with DLR ranging from 0.14 to 0.4 were collected to analyze the correlation between SNP calling failure rate and DLR.

The SNP alleles in the Agilent CGH + SNP arrays locate in the recognition site AGCT by restriction enzyme Alu I and GTAC by RsaI. Polymerase chain reaction (PCR)based restriction enzyme digestion and Sanger sequencing were used to assess the accuracy of SNP calling by Agilent CGH + SNP arrays. Five SNPs (rs3844608, rs8060511, rs8062853, rs216166 and rs10871449) were chosen and PCR primer pairs for each SNP were designed using Primer Express 3.0 (Additional file 1: Table S1). SNP calling by aCGH from 25 cases with DLR $<2.0$ were further verified by fragment pattern from the digestion of PCR products by AluI or RsaI. When the SNP calling and enzyme digestion pattern showed inconsistent results, the allelic patterns were further analyzed using Sanger sequencing of the PCR product. Routine PCR was performed according to a standardized protocol for native Taq DNA polymerase (Invitrogen). Fragment analysis was run on Agilent 2100 Bioanalyzer (Agilent Technologies, Inc., Santa Clara, CA). Sanger sequencing was performed by submitting purified PCR products to the Yale Center of Genomic Analysis.

\section{Assessing the distribution of $\mathrm{ROH}$ in case series}

An Excel file was used to collect data from each case series. The input data for each case included an anonymous case number, gender, age, sample type, clinical indication, aCGH and chromosome results, microarray platform, DLR measurement, and $\mathrm{ROH}$ detected. The number of $\mathrm{ROH}$, the number of SNP probes hybridized to each $\mathrm{ROH}$, the $\mathrm{ROH}$ size defined by genomic coordinates, and the chromosomal G-band location of $\mathrm{ROH}$ for each case were recorded. The numbers and sizes of $\mathrm{ROH}$ were compared among the four case series. To assess the chromosomal distribution of $\mathrm{ROHs}$, the observed numbers and sizes of $\mathrm{ROH}$ and adjusted numbers and sizes by the genomic portion of each chromosome were analyzed. Frequencies of recurrent $\mathrm{ROH}$ at specific chromosomal loci were calculated by the number of cases with the recurrent $\mathrm{ROH}$ divided by total number of cases. The database Progenetix was used for data conversion and analysis [19]. The chromosomal location and genomic coordinate of each $\mathrm{ROH}$ were entered into the database and converted into schematic views that allowed a visualization and comparison of $\mathrm{ROH}$ frequencies.

\section{Statistics}

One-way ANOVA analysis was used to compare the difference of SNP calling failure rate among samples with different ranges of DLR (http://vassarstats.net/anovalu.html). Chisquare analysis was performed to compare the difference among the case series for the number and size distribution of $\mathrm{ROH}$ by using the webtool: Chi-square Test Calculator (http://www.socscistatistics.com/tests/chisquare2/ Default2.aspx).

\section{Results}

\section{Analytical validity of aCGH on detecting ROH}

The density of SNP probes (defined by the number of probes per $\mathrm{Mb}$ ) varied from 3 to 59 of detected $\mathrm{ROH}$. The average number of SNP probes per $\mathrm{Mb}$ is 18 for both platforms which set analytical resolution arbitrarily as $1 \mathrm{Mb}$. The data of SNP calling on each SNP probe run by Agilent array $180 \mathrm{~K}$ and $400 \mathrm{~K}$ were exported from 30 cases with a range of DLR from 0.14 to 0.4 . A total of 59,645 SNP probes were exported for each case run by the Agilent $180 \mathrm{~K}$ array, and 64,366 SNP probes for the $400 \mathrm{~K}$ array. The 30 cases were divided into three groups by DLR range of below 0.2, 0.2-0.3, and above 
0.3. Of the 30 cases ran by $180 \mathrm{~K}$, the mean SNP calling failure rate (NN calling rate) for 10 cases with DLR below 0.2 was $28.2 \%$, for 15 cases with DLR range 0.2 to 0.3 was $36.5 \%$, and for five cases with DLR above 0.3 was $38.5 \%$ (Fig. 1a). Of the 30 cases run by the $400 \mathrm{k}$ array, the mean calling failure rate for 12 cases with DLR below 0.2 was $25.2 \%$, for 11 cases with DLR range 0.2 to 0.3 was $35.3 \%$, for seven cases with DLR above 0.3 was $37 \%$ (Fig. 1b). Statistical analysis showed significant difference between DLR $<0.2$ and $0.2-0.3$ or DLR $>0.3$ $(p<0.01)$, but no difference between the DLR of $0.2-0.3$ and DLR $>0.3$ on both platforms. This result indicated that the DLR below 0.2 should be used as a quality control parameter to ensure consistency of SNP calling.

PCR was performed for the five selected SNP alleles on 25 cases with DLR below 0.2. Of these 125 SNPs tested, four SNP calling could not be confirmed by either PCR-restriction digestion or Sanger sequencing due to poor PCR amplification and were excluded. Of the remaining 121 SNPs, 112 SNPs showed consistent results from both aCGH SNP calling and direct PCR restriction enzyme digestion, but nine SNPs showed inconsistent results and were proved as false by Sanger sequencing on PCR products. The results of SNP callings from aCGH and patterns of PCR restriction enzyme digestion and Sanger sequencing are listed in Additional file 1: Table $\mathrm{S} 2$ and two verified false SNP callings by aCGH are shown in Fig. 2. Of the five SNPs tested, the accuracy of SNP calling by aCGH is $93 \%$ (112/ 121). It was noted that false callings occurred all in the two SNP alleles rs3844608 (four false in 25 callings) and rs8060511 (five false in 24 callings) but was not observed in the other three SNP alleles rs8062853, rs216166 and rs10871449. This observation suggested that some SNP loci may be prone to incomplete restriction enzyme digestion and induced false calling.

\section{Distribution and frequency of ROH Numbers and sizes of $\mathrm{ROH}$}

A total of 958 cases were included in the study over the four years from 2014 to 2017. 142 were parental, 500 were postnatal/pediatric, 195 were prenatal CVS, and 121 were prenatal AF cases. All of them had normal karyotype and aCGH results. Cases with no $\mathrm{ROH}$ were detected in $31.7 \%$ of parental, $32.8 \%$ of pediatric, $38.5 \%$ of CVS, and $36.4 \%$ of AF cases. Cases with one $\mathrm{ROH}$ were detected in $29.6 \%$ of parental, $33.6 \%$ of pediatric, $33.9 \%$ of CVS, and $32.7 \%$ of AF cases. Cases with two $\mathrm{ROH}$ were detected $23.2 \%$ of parental, $18.4 \%$ of pediatric, $17.4 \%$ of CVS, and $23.1 \%$ of AF cases. Cases with three $\mathrm{ROH}$ were detected in $8.5 \%$ of parental, $8.2 \%$ of pediatric, $3.6 \%$ of CVS, and $5.8 \%$ of AF cases. Cases with four $\mathrm{ROH}$ were detected in $2.1 \%$ of parental, $4.0 \%$ of pediatric, $3.6 \%$ of CVS, and $1.7 \%$ of AF cases. Cases with five or more $\mathrm{ROH}$ were detected in $4.9 \%$ of parental, $3.0 \%$ of pediatric, $3.1 \%$ of CVS, and $2.5 \%$ of AF cases. There was no significant difference among the parental, pediatric, and prenatal case series, and no differences amongst the different tissue types (Additional file 1: Table S3, Fig. 3a). Therefore, of the 958 cases from the four-tested case series, the proportion of cases possessing no $\mathrm{ROH}$, one to four $\mathrm{ROH}$, and five or more $\mathrm{ROH}$ accounted for 34.2, 62.5, and $3.2 \%$ of cases, respectively (Fig. 3b).

The sizes of detected $\mathrm{ROH}$ varied from $1 \mathrm{Mb}$ to 20 $\mathrm{Mb}$. $\mathrm{ROH}$ less than $5 \mathrm{Mb}$ in size were noted in $73 \%$ of parental, $77 \%$ of pediatric, $74 \%$ of CVS, and $76 \%$ of $\mathrm{AF}$ case series, respectively. $\mathrm{ROH}$ less than $10 \mathrm{Mb}$ in size
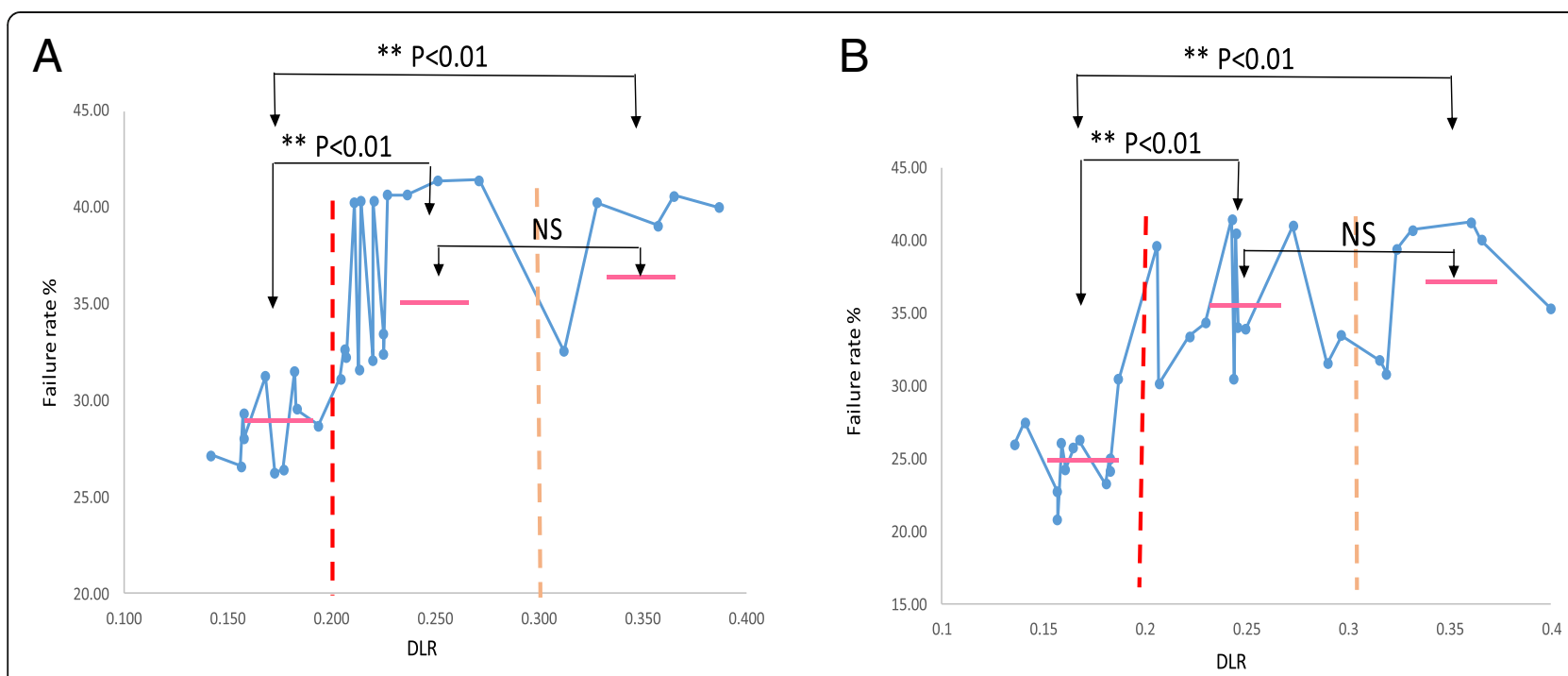

Fig. 1 SNP calling failure rate in association with DLR. SNP calling data were extracted from a. Agilent $180 \mathrm{~K}$ aCGH/SNP array, and b. Agilent $400 \mathrm{~K}$ aCGH/SNP array. SNP calling failure rate increased significantly when DLR is greater than $0.2(* *, p<0.01)$. No significant difference (NS) between samples of DLR $0.2-0.3$ and $D L R>0.3$ 

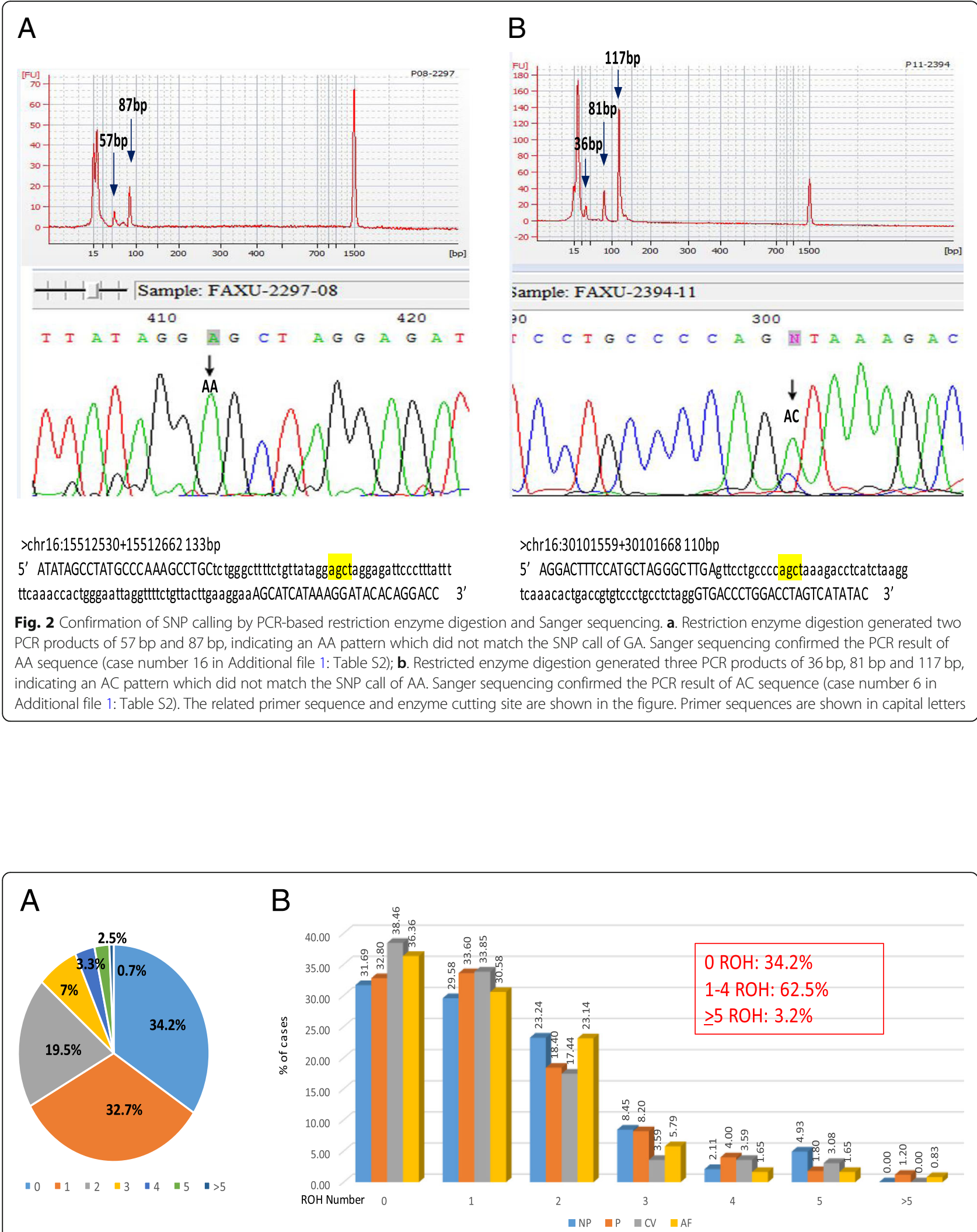

Fig. 3 Distribution of $\mathrm{ROH}$ in the tested cases. a. A pie chart showing the percentage of different $\mathrm{ROH}$ counts $(0,1,2,3,4,5$, and $>5)$ in the total of 958 tested subjects. b. A bar graph showing the comparison of ROH counts in different case series shown by different color (blue for NP, normal parents; orange for $\mathrm{P}$, pediatric cases; grey for CV, prenatal CVS cases; and yellow for AF, prenatal AF cases) 
were noted in $96 \%$ of parental, $94 \%$ of pediatric, $98 \%$ of CVS, and $97 \%$ of AF case series, respectively. The size distribution showed no significant differences among the four series (Fig. 4a). This result indicated that a cutoff value of $10 \mathrm{Mb}$ can exclude more than $95 \%$ of $\mathrm{ROH}$ observed in normal cases.

\section{Chromosomal location and recurrence of $\mathrm{ROH}$ in the genome}

Of the $1196 \mathrm{ROH}$ detected in the tested series, the sum of $\mathrm{ROH}$ sizes was $5163.3 \mathrm{Mb}$ and the numbers and sizes of $\mathrm{ROH}$ distributed in each chromosome varied. The number of ROH observed ranged from 5 to 8 for chromosomes 15, 16, 19, 21 and 22, and from 18 to 150 for the other chromosomes. The average sizes of $\mathrm{ROH}$ ranged from $<3 \mathrm{Mb}$ on chromosomes 9 and 16 to $>6$ $\mathrm{Mb}$ on chromosomes 17, 19, 22 and X; the rest of the chromosomes had an average $\mathrm{ROH}$ size close to $4 \mathrm{Mb}$ (Fig. 4b). Assuming a random distribution of $\mathrm{ROH}$ in each chromosome, the expected numbers and sizes of $\mathrm{ROH}$ on each chromosome were calculated per the actual portion of each chromosome in the whole genome times the total number or size of the detected $\mathrm{ROH}$. Table 1 summarized the observed and expected numbers and sizes of $\mathrm{ROH}$ in each chromosome and showed a non-random distribution. Chromosomes 2, 6, 8, 10, 11 and 12 showed significant over-representation of both size and number of $\mathrm{ROH}$, while chromosomes $3,7,14,15,16$, $17,19,20,21$ and 22 showed under-representation. $\mathrm{ROH}$ was most prevalent in chromosome $8(150 / 1196,12.5 \%)$. The second most common location is in chromosome 2 (132/1196, 11.0\%), followed by chromosomes 6 (98/1196, 8.2\%), 10 (85/1196, 7.1\%), 12 (76/1196, 6.4\%), 4 (73/1196, 6.1\%), 5 (73/1196, 6.1\%), 11 (71/1196, 5.9\%), 1 (68/1196,

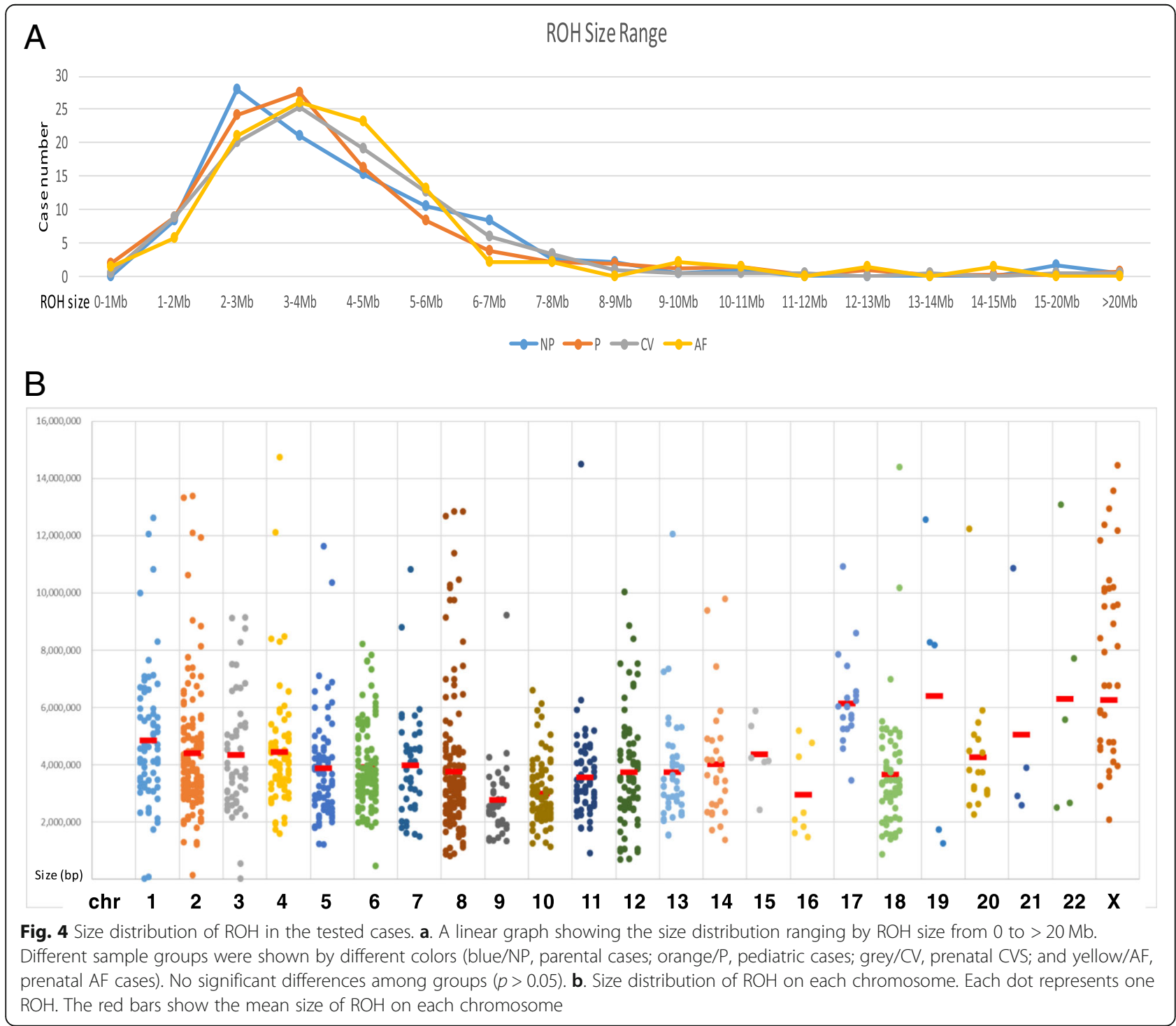


Table 1 Chromosomal distribution of $\mathrm{ROH}$

\begin{tabular}{|c|c|c|c|c|c|c|}
\hline Chromosome & Chromosome size (bp) & $\%$ of genome & Observed $\mathrm{ROH}$ size $(\mathrm{Mb})$ & Expected $\mathrm{ROH}$ size $(\mathrm{Mb})$ & Observed $\mathrm{ROH}$ No. & Expected $\mathrm{ROH}$ No \\
\hline 1 & $247,199,719$ & 8 & 339.2 & 258.2 & 68 & 96 \\
\hline 2 & $242,751,149$ & 7.9 & 601.4 & 413.1 & 132 & 94 \\
\hline 3 & $199,446,827$ & 6.5 & 230.0 & 407.9 & 52 & 78 \\
\hline 4 & $191,263,063$ & 6.2 & 334.9 & 335.6 & 73 & 74 \\
\hline 5 & $180,837,866$ & 5.9 & 288.0 & 320.1 & 73 & 71 \\
\hline 6 & $170,896,993$ & 5.5 & 420.3 & 304.6 & 98 & 66 \\
\hline 7 & $158,821,424$ & 5.2 & 159.1 & 284.0 & 40 & 62 \\
\hline 8 & $146,274,826$ & 4.7 & 567.8 & 268.5 & 150 & 56 \\
\hline 9 & $140,442,298$ & 4.6 & 110.7 & 242.7 & 40 & 55 \\
\hline 10 & $135,374,737$ & 4.4 & 267.3 & 237.5 & 85 & 53 \\
\hline 11 & $134,452,384$ & 4.4 & 245.8 & 227.2 & 71 & 53 \\
\hline 12 & $132,289,534$ & 4.3 & 302.5 & 227.2 & 76 & 51 \\
\hline 13 & $114,127,980$ & 3.7 & 180.7 & 222.0 & 43 & 44 \\
\hline 14 & $106,360,585$ & 3.5 & 112.8 & 191.0 & 27 & 42 \\
\hline 15 & $100,338,915$ & 3.3 & 26.2 & 180.7 & 5 & 39 \\
\hline 16 & $88,822,254$ & 2.9 & 23.6 & 170.4 & 8 & 35 \\
\hline 17 & $78,654,742$ & 2.6 & 128.9 & 149.7 & 21 & 31 \\
\hline 18 & $76,117,153$ & 2.5 & 231.6 & 134.2 & 59 & 30 \\
\hline 19 & $63,806,651$ & 2.1 & 32.0 & 129.1 & 5 & 25 \\
\hline 20 & $62,435,965$ & 2 & 76.8 & 108.4 & 18 & 24 \\
\hline 21 & $46,944,323$ & 1.5 & 43.8 & 103.3 & 5 & 18 \\
\hline 22 & $49,528,953$ & 1.6 & 31.6 & 77.4 & 5 & 19 \\
\hline$x$ & $154,913,754$ & 5 & 408.4 & 82.6 & 42 & 60 \\
\hline Total & $3,079,843,747$ & & 5163.3 & & 1196 & \\
\hline
\end{tabular}

5.7\%), and 18 (59/1196, 4.9\%). ROH was less likely to be seen in chromosome 16 with a frequency of $0.7 \%$ and was least likely to be observed in chromosomes $15,19,21$, and 22 with a frequency of $0.4 \%$.

Chromosomal regions harboring overlapping $\mathrm{ROH}$ in different cases were identified in the tested series. Those overlapping $\mathrm{ROH}$ with a frequency larger than $0.5 \%$ were considered as recurrent $\mathrm{ROH}$ in the genome. Of the total of $1196 \mathrm{ROHs}, 47$ chromosome regions of overlapping $\mathrm{ROH}$ were noted with a recurrent frequency of larger than $0.5 \%$ and 17 chromosomal regions of $\mathrm{ROH}$ were noted with a recurrent frequency larger than $1 \%$ (Additional file 1: Table S4). Of those 17 regions of recurrent ROH, 2p12p11.2, 8q11.1q12.1, 11p13p12, $12 \mathrm{p} 11.1 \mathrm{q} 12$, and $18 \mathrm{q} 11.2 \mathrm{q} 12.1$ are in the pericentric regions. Figure 5 showed the chromosomal regions with recurrent $\mathrm{ROH}$. The most frequent regions harboring recurrent ROHs were 8q11.1q12.1 (chr8:46,940,022-58, 936,071), 11p13p12 (chr11:35,538,690-41,944,671), and 10q21.1q21.2 (chr10:53,569,925-62,598,787) with a frequency of $4.2 \%, 3.6 \%$ and $3.2 \%$, respectively. All recurrent $\mathrm{ROH}$ were observed in the pericentric regions or interstitial regions but not in the subtelomeric regions.

\section{Discussion}

Implementation of CGH + SNP microarrays have enabled simultaneous interrogation of genomic DNA for $\mathrm{CNV}$ and copy neutral $\mathrm{ROH}$. Multiple microarray platforms exist and vary in the placement and density of the SNP probes. Laboratories that perform this microarray analysis should establish quality control and quality assurance measures and reference values for each platform in the technical procedures and reporting practices. In this study, we proved that the current Agilent CGH + SNP $180 \mathrm{~K}$ and $400 \mathrm{~K}$ microarray platforms showed a consistent 73-75\% SNP calling rate with DLR below 0.2, and the accuracy of SNP calling was approximately $93 \%$. The SNP calling rate decreased significantly to $60-70 \%$ when the DLR ratio was greater than 0.2 and 0.3 . According to the manufacturer's quality control metrics parameters, DLR $<0.2$ was considered as excellent, DLR in the range of 0.2-0.3 was considered as a "good run" while DLR greater than 0.3 was classified as "to be evaluated". The SNP calling rate showed no difference from the DLR $0.2-0.3$ and greater than 0.3 but a clear difference from DLR below 0.2. Therefore, to ensure consistency and accuracy in SNP calling, DLR below 0.2 should be used a 

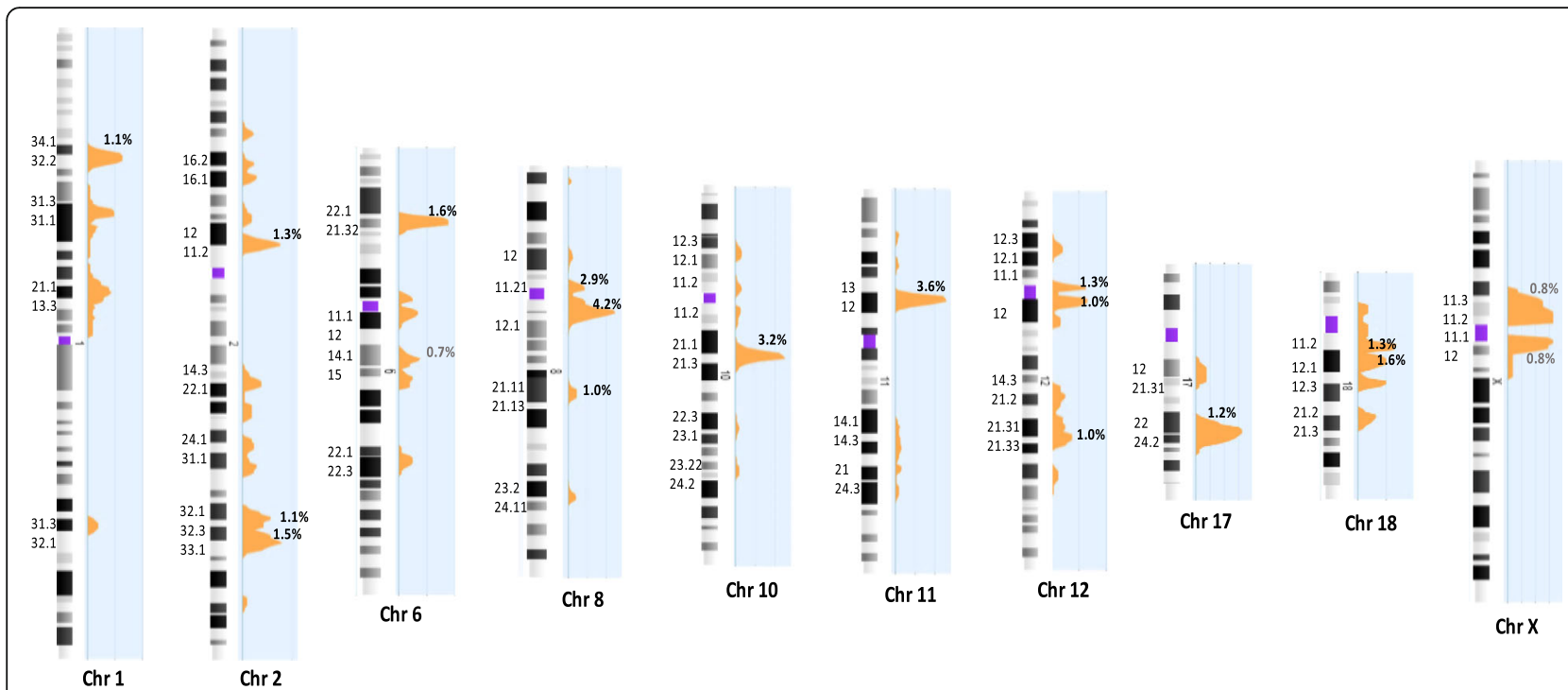

Fig. 5 Schematic view of chromosomal regions and frequencies of the recurrent $\mathrm{ROH}$. Recurrent $\mathrm{ROH}$ regions with a frequency greater than $1 \%$ are shown

cutoff value for reporting $\mathrm{ROH}$. In practice, it is reasonable that DLR below 0.2 for current microarray platforms can be considered as a reliable tool for screening of $\mathrm{ROH}$ and reporting of $\mathrm{CNV}$ and $\mathrm{ROH}$. When the DLR is $0.2 \sim 0.3$, the SNP calling could be used to confirm CNV reading but only the $\mathrm{CNV}$ is to be reported, and when the DLR $>0.3$, the case should be repeated.

It has been recognized that the detection of one or more $\mathrm{ROH}$ is not an abnormal finding in apparently normal individuals of an outbred population. $\mathrm{ROH}$ arise from the two brought-together ancestral haplotypes. Longer haplotypes are inherited from recent common ancestors whereas shorter haplotypes are inherited from distant ones. Different population histories give rise to divergent distributions of long and short $\mathrm{ROH}$ [6]. Although the numbers and sizes of $\mathrm{ROH}$ vary dramatically among published data, most of the studies showed that normal outbred populations rarely have $\mathrm{ROH}$ above $10 \mathrm{Mb}$ and commonly have smaller $\mathrm{ROH}$ of less than $4 \sim 5 \mathrm{Mb}[20,21]$. This occurs across all populations and is termed ancestral $\mathrm{ROH}$. Our results from a total of 958 tested cases showed that $34 \%$ of cases had no $\mathrm{ROH}, 66 \%$ had at least one $\mathrm{ROH}$, and $94 \%$ of this tested population had less than $3 \mathrm{ROH}$. Of the $1196 \mathrm{ROH}$ detected, $70 \%$ of them had a size of less than $5 \mathrm{Mb}$ and $95 \%$ of $\mathrm{ROH}$ were less than $10 \mathrm{Mb}$. These results were consistent with the published data.

$\mathrm{ROH}$ distribution over the chromosomes was found to be nonrandom in mammals, including humans [21-24]. $\mathrm{ROH}$ are more common in regions of high linkage disequilibrium (LD) and low recombination [5] as well as regions of low genetic diversity [21]. In our study, the most frequent region with $\mathrm{ROH}$ is the pericentromeric region of chromosome 8 . Indeed, most of the regions that harbor a recurrent $\mathrm{ROH}$ are pericentromeric regions, which in theory should have a low recombination rate. Furthermore, the larger average size on the $\mathrm{X}$ chromosome also indicated low recombination frequency on the $\mathrm{X}$ chromosome through generations. Most chromosomes have a similar average $\mathrm{ROH}$ size of close to $4 \mathrm{Mb}$, so this may reflect the fact that there are no marked differences among chromosomes in the concentration of recombination hotspots [25]. Our data reported that, among the $1196 \mathrm{ROH}$ greater than $1 \mathrm{Mb}$, seventeen overlapped regions were recurrently identified with a frequency of greater than $1 \%$. The presence of recurrent $\mathrm{ROH}$ in the outbred population indicated that these $\mathrm{ROH}$ may represent an ancestral $\mathrm{ROH}$ with insignificant or limited clinical implications.

In our study, some chromosomes showed a very low frequency in presenting an $\mathrm{ROH}$, such as chromosomes $15,16,19,21$ and 22 . The underlying mechanism is unclear. Populations with both reduced effective population size in the past and recent inbreeding have the greatest burden of $\mathrm{ROH}$; on the contrary, inbreeding depression could be a cause of eliminating the $\mathrm{ROH}$ in a certain chromosome. Studies showed that $\mathrm{ROH}$ are more enriched for homozygous deleterious variants than for non-deleterious variants [26], so that $\mathrm{ROH}$ are important reservoirs of homozygous deleterious variations [27]. In theory, inbreeding increases the chance of rare variants (including deleterious variants) to be in a homozygous state, and if the recessive variants are fatal, the inbreeding will in fact purge the recessive alleles from the population. Over time, this may result in fewer $\mathrm{ROH}$ in certain regions on certain chromosomes. We do not know if this could explain our findings, and it would be worthwhile to further investigate the rare, deleterious and fatal recessive variants on these chromosomes. 
The cumulative extent of $\mathrm{ROH}$ in any given case is determined by multiple factors such as parental relatedness, ethnicity, recombination events, and chromosomal aberrations. The significance of such findings and deriving clues towards molecular testing and genotype-phenotype correlations necessitates critical evaluation of the microarray data in each individual case [28]. Wierenga et al. [29] reported variance in total $\mathrm{ROH}$ based on different size thresholds and illustrated the importance of threshold determination. The variation in cutoff values of $\mathrm{ROH}$ reported in the literature was reflected in the research laboratory's reporting policy. In 2013, ACMG published a guideline regarding the documentation of suspected consanguinity by genomic testing [16]. From the size distribution detected by the current Agilent $\mathrm{CGH}+\mathrm{SNP}$ array platforms, $10 \mathrm{Mb}$ is a reasonable cutoff level to report the incidental finding of a single $\mathrm{ROH}$. In addition, the recurrent $\mathrm{ROH}$ identified in our study indicated that these $\mathrm{ROH}$ may represent an ancestral $\mathrm{ROH}$ and should be considered as a reference set of "benign or likely benign polymorphic" $\mathrm{ROH}$ and should not be included in the reports. However, caution should be applied to the gene content especially disease-causing dominant or recessive gene mutations within these recurrent $\mathrm{ROH}$. These cutoff settings should avoid missing important clinical information that may impart significant clinical consequences, but at the same time minimize the chance of overestimating it which may cause dramatic burden for the clinicians and genetic counselors, as well as to the patient's family, both emotionally and financially.

\section{Conclusion}

In the past decade, microarray testing for the assessment of genomic copy number imbalances in clinical laboratories has grown quickly. The detection of UPD, consanguinity, and isolated $\mathrm{ROH}$ has been an integral part of genomic analysis using many available platforms. The present study assessed the consistency and accuracy of SNP calling with the current Agilent CGH + SNP platforms and observed a nonrandom chromosomal distribution of $\mathrm{ROH}$ from parental, pediatric and prenatal case series. Based on the reference values of DLR below $0.2,75 \%$ of SNP calling rate, and $93 \%$ accuracy of SNP calling, the CGH + SNP $180 \mathrm{~K}$ and $400 \mathrm{~K}$ platforms could be considered as a reliable method to screen for $\mathrm{ROH}$ larger than $10 \mathrm{Mb}$. If clinically indicated, correlating gene content of $\mathrm{ROH}$ with mutation testing results and patient phenotypes should be recommended.

\section{Additional file}

Additional file 1: Table S1. PCR primer for selected SNPS. Table S2.

Confirmation of SNP calling by PCR/Sequencing. Table S3. $\mathrm{ROH}$

distribution. Table S4. Frequencies of recurrent $\mathrm{ROH}$. (DOCX 78 kb)

\section{Abbreviations}

aCGH: Array comparative genomic hybridization; AF: Amniotic fluid CNV: Copy number variants; CVS: Chorionic villi samples; DLR: Derivative
Log2 Ratio; IBD: Identical by descent; LD: Linkage disequilibrium; $\mathrm{ROH}$ : Regions of homozygosity; SNP: Single nucleotide polymorphism; UPD: Uniparental disomy

\section{Acknowledgements}

The authors like to thank Ms. Audrey Meusel for proofreading this manuscript.

\section{Funding}

Not applicable.

\section{Availability of data and materials}

The datasets used and/or analyzed during the current study are available from the corresponding author on reasonable request.

Authors' contributions

ZX, WW: initial validation of SNP calling by PCR; KC, KA, AD, BG: UConn Project on SNP., FX, HCH: Data collection and processing; JW, PL: Organizing project, analyzing data and drafting manuscript. All authors have approved the final article.

Ethics approval and consent to participate

Not applicable.

Consent for publication

Not applicable.

Competing interests

None to disclose.

\section{Publisher's Note}

Springer Nature remains neutral with regard to jurisdictional claims in published maps and institutional affiliations.

\section{Author details}

${ }^{1}$ Department of Genetics, Yale University School of Medicine, New Haven, CT 06520, USA. ²Diagnostic Genetics Program, University of Connecticut, Storrs, CT 06269, USA. ${ }^{3}$ Shenzhen Maternity and Child Healthcare Hospital,

Shenzhen, Guangdong, China. ${ }^{4}$ PreventionGenetics, Marshfield, WI 54449, USA.

Received: 29 January 2019 Accepted: 19 February 2019

Published online: 06 March 2019

\section{References}

1. Broman KW, Weber JL. Long homozygous chromosomal segments in reference families from the centre d'Etude du polymorphisme humain. Am J Hum Genet 1999;65(6):1493-1500 doi: https://doi.org/10.1086/ 302661[published Online First: Epub Date]|.

2. Sund $\mathrm{KL}$, Rehder CW. Detection and reporting of homozygosity associated with consanguinity in the clinical laboratory. Hum Hered 2014;77(1-4):217-224 doi: https://doi.org/10.1159/000362448[published Online First: Epub Date]|.

3. Fan YS, Ouyang $X$, Peng J, et al. Frequent detection of parental consanguinity in children with developmental disorders by a combined CGH and SNP microarray. Mol Cytogenet 2013;6(1):38 doi: https://doi.org/10. 1186/1755-8166-6-38[published Online First: Epub Date]|.

4. Kearney HM, Kearney JB, Conlin LK. Diagnostic implications of excessive homozygosity detected by SNP-based microarrays: consanguinity, uniparental disomy, and recessive single-gene mutations. Clin Lab Med 2011;31(4):595-613 doi: https://doi.org/10.1016/j.cll.2011.08.003[published Online First: Epub Date]l.

5. Gibson J, Morton NE, Collins A. Extended tracts of homozygosity in outbred human populations. Hum Mol Genet 2006;15(5):789-795 doi: https://doi. org/10.1093/hmg/ddi493[published Online First: Epub Date]|.

6. Ceballos FC, Joshi PK, Clark DW, et al. Runs of homozygosity: windows into population history and trait architecture. Nat Rev Genet 2018;19(4):220-34 doi: https://doi.org/10.1038/nrg.2017.109[published Online First: Epub Date]|.

7. Pemberton TJ, Absher D, Feldman MW et al. Genomic patterns of homozygosity in worldwide human populations. Am J Hum Genet 2012; 
91(2):275-292 doi: https://doi.org/10.1016/j.ajhg.2012.06.014[published Online First: Epub Date].

8. Xiang B, Li A, Valentin D, et al. Analytical and clinical validity of wholegenome oligonucleotide array comparative genomic hybridization for pediatric patients with mental retardation and developmental delay. Am J Med Genet A 2008;146A(15):1942-1954 doi: https://doi.org/10.1002/ajmg.a 32411[published Online First: Epub Date]|.

9. Xiang B, Zhu H, Shen Y, et al. Genome-wide oligonucleotide array comparative genomic hybridization for etiological diagnosis of mental retardation: a multicenter experience of 1499 clinical cases. J Mol Diagn 2010;12(2):204-212 doi: https://doi.org/10.2353/jmoldx.2010. 090115[published Online First: Epub Date]|.

10. Miller DT, Adam MP, Aradhya S, et al. Consensus statement: chromosomal microarray is a first-tier clinical diagnostic test for individuals with developmental disabilities or congenital anomalies. Am J Hum Genet 2010; 86(5):749-764 doi: https://doi.org/10.1016/j.ajhg.2010.04.006[published Online First: Epub Date].

11. Manning M, Hudgins L, Professional P, et al. Array-based technology and recommendations for utilization in medical genetics practice for detection of chromosomal abnormalities. Genet Med 2010;12(11):742-745 doi: https:// doi.org/10.1097/GIM.0b013e3181f8baad[published Online First: Epub Date].

12. Schaaf CP, Wiszniewska J, Beaudet AL. Copy number and SNP arrays in clinical diagnostics. Annu Rev Genomics Hum Genet 2011;12:25-51 doi: https://doi.org/10.1146/annurev-genom-092010-110715[published Online First: Epub Datel|.

13. Shaffer $L G$, Rosenfeld JA. Microarray-based prenatal diagnosis for the identification of fetal chromosome abnormalities. Expert Rev Mol Diagn 2013;13(6):601-611 doi: https://doi.org/10.1586/14737159.2013. 811912[published Online First: Epub Date].

14. Wei Y, Xu F, Li P. Technology-driven and evidence-based genomic analysis for integrated pediatric and prenatal genetics evaluation. J Genet Genomics 2013;40(1):1-14 doi: https://doi.org/10.1016/j.jgg.2012.12.004[published Online First: Epub Date]|.

15. Zhou Q, Wu SY, Amato K, et al. Spectrum of Cytogenomic Abnormalities Revealed by Array Comparative Genomic Hybridization on Products of Conception Culture Failure and Normal Karyotype Samples. J Genet Genomics 2016;43(3):121-131 doi: https://doi.org/10.1016/j.jgg.2016.02 002[published Online First: Epub Date]|.

16. Rehder CW, David KL, Hirsch B, et al. American College of Medical Genetics and Genomics: standards and guidelines for documenting suspected consanguinity as an incidental finding of genomic testing. Genet Med 2013: 15(2):150-2 doi: https://doi.org/10.1038/gim.2012.169[published Online First: Epub Date].

17. Grote L, Myers M, Lovell A, et al. Variability in laboratory reporting practices for regions of homozygosity indicating parental relatedness as identified by SNP microarray testing. Genet Med 2012;14(12):971-976 doi: https://doi.org/ 10.1038/gim.2012.83[published Online First: Epub Date]].

18. Xiang B, Li P, Hemingway SS, et al. CytoAccess, a relational laboratory information management system for a clinical cytogenetics laboratory. J Assoc Genet Technol. 2006;32(4):168-70.

19. Baudis M, Cleary ML. Progenetix.net: an online repository for molecular cytogenetic aberration data. Bioinformatics. 2001;17(12):1228-9.

20. McQuillan R, Leutenegger AL, Abdel-Rahman R, et al. Runs of homozygosity in European populations. Am J Hum Genet 2008;83(3):359-372 doi: https:// doi.org/10.1016/j.ajhg.2008.08.007[published Online First: Epub Date]|.

21. Nothnagel M, Lu TT, Kayser M, et al. Genomic and geographic distribution of SNP-defined runs of homozygosity in Europeans. Hum Mol Genet 2010 19(15):2927-2935 doi: https://doi.org/10.1093/hmg/ddq198[published Online First: Epub Date].

22. Bosse $\mathrm{M}$, Megens $\mathrm{HJ}$, Madsen $\mathrm{O}$, et al. Regions of homozygosity in the porcine genome: consequence of demography and the recombination landscape. PLoS Genet 2012;8(11):e1003100 doi: https://doi.org/10.1371/ journal.pgen.1003100[published Online First: Epub Date]|.

23. Curtis D, Vine AE, Knight J. Study of regions of extended homozygosity provides a powerful method to explore haplotype structure of human populations. Ann Hum Genet 2008;72(Pt 2):261-278 doi: https://doi.org/10. 1111/j.1469-1809.2007.00411.x [published Online First: Epub Date]|

24. Kirin M, McQuillan R, Franklin CS, et al. Genomic runs of homozygosity record population history and consanguinity. PLoS One 2010;5(11):e13996 doi: https://doi.org/10.1371/journal.pone.0013996[published Online First: Epub Date].
25. International HapMap C, Frazer KA, Ballinger DG, et al. A second generation human haplotype map of over 3.1 million SNPs. Nature 2007:449(7164):851861 doi: https://doi.org/10.1038/nature06258[published Online First: Epub Date]|.

26. Szpiech ZA, Xu J, Pemberton TJ, et al. Long runs of homozygosity are enriched for deleterious variation. Am J Hum Genet 2013;93(1):90-102 doi: https://doi.org/10.1016/j.ajhg.2013.05.003[published Online First: Epub Date]|.

27. Alsalem AB, Halees AS, Anazi $S$, et al. Autozygome sequencing expands the horizon of human knockout research and provides novel insights into human phenotypic variation. PLoS Genet 2013;9(12):e1004030 doi: https:// doi.org/10.1371/journal.pgen.1004030[published Online First: Epub Date]|.

28. Wang JC, Ross L, Mahon LW, et al. Regions of homozygosity identified by oligonucleotide SNP arrays: evaluating the incidence and clinical utility. Eur J Hum Genet 2015;23(5):663-71 doi: https://doi.org/10.1038/ejhg.2014. 153[published Online First: Epub Date].

29. Wierenga KJ, Jiang Z, Yang AC, et al. A clinical evaluation tool for SNP arrays, especially for autosomal recessive conditions in offspring of consanguineous parents. Genet Med 2013;15(5):354-360 doi: https://doi. org/10.1038/gim.2012.136[published Online First: Epub Date]|.

\section{Ready to submit your research? Choose BMC and benefit from:}

- fast, convenient online submission

- thorough peer review by experienced researchers in your field

- rapid publication on acceptance

- support for research data, including large and complex data types

- gold Open Access which fosters wider collaboration and increased citations

- maximum visibility for your research: over $100 \mathrm{M}$ website views per year

At BMC, research is always in progress.

Learn more biomedcentral.com/submissions 